\title{
Perceptual learning in auditory temporal discrimination: No evidence for a cross-modal transfer to the visual modality
}

\author{
EINAT LAPID AND ROLF ULRICH \\ University of Tübingen, Tübingen, Germany \\ AND \\ THOMAS RAMMSAYER \\ University of Bern, Bern, Switzerland
}

\begin{abstract}
Perceptual learning was used to study potential transfer effects in a duration discrimination task. Subjects were trained to discriminate between two empty temporal intervals marked with auditory beeps, using a twoalternative forced choice paradigm. The major goal was to examine whether perceptual learning would generalize to empty intervals that have the same duration but are marked by visual flashes. The experiment also included longer intervals marked with auditory beeps and filled auditory intervals of the same duration as the trained interval, in order to examine whether perceptual learning would generalize to these conditions within the same sensory modality. In contrast to previous findings showing a transfer from the haptic to the auditory modality, the present results do not indicate a transfer from the auditory to the visual modality; but they do show transfers within the auditory modality.
\end{abstract}

Humans and animals possess the impressive cognitive ability of temporal discrimination, and this capability appears to be fundamental to perception and action (Buhusi \& Meck, 2005; Ivry \& Spencer, 2004; Mauk \& Buonomano, 2004). Despite temporal cognition being a basic mental ability, there is still controversy surrounding the mechanisms that underlie this ability.

One theoretical account of human timing assumes the existence of a single internal clock (cf. Grondin, 2003; Rammsayer \& Ulrich, 2001). The internal clock mechanism is characterized by an accumulator that registers neural pulses that occur during a certain time interval. Thus, the number of pulses registered during this interval is an index of the perceived duration of this interval. A second account, the entrainment theory, holds that human timing involves an internal oscillator that dynamically synchronizes attention with external events (e.g., Jones, 1976; Jones \& Boltz, 1989; Large \& Jones, 1999; McAuley \& Jones, 2003). This adaptive oscillator induces expectancies when a stimulus ends, and thus enables a person to judge whether an attended temporal event fits to a previously encountered one. Although both accounts postulate essentially different timing mechanisms, they share the assumption that the postulated mechanisms can operate on different sensory inputs - that is, the same neural circuit is involved in temporal discrimination, even when it receives information from different modalities (e.g., Ulrich, Nitschke, \& Rammsayer, 2006). According to a third account, however, distributed mechanisms underlie human timing. This notion assumes that various brain areas - and, thus, different circuits-underlie temporal discriminations (Buhusi \& Meck, 2005; Lewis \& Miall, 2003). Although these mechanisms may receive inputs from different sensory modalities (Mauk \& Buonomano, 2004), they may operate only within specific time ranges (e.g., Bendixen, Grimm, \& Schröger, 2005; Karmarkar \& Buonomano, 2007; Rammsayer \& Lima, 1991). For example, one such circuit may operate within a range of milliseconds, whereas another circuit may operate within a range of seconds (Lewis \& Miall, 2003).

Several researchers have employed perceptual learning in order to examine whether range-specific timing mechanisms exist. For example, subjects in the study by Wright, Buonomano, Mahncke, and Merzenich (1997) were presented in each trial with two temporal intervals and were asked to indicate the longer of the two intervals in a two-alternative forced choice (2AFC) task. These authors measured the difference limen (DL) to assess temporal discrimination performance of their subjects. Subjects practiced for 10 days on the discrimination of an empty, 100 -msec interval that was marked by two beeps of the same frequency (e.g., $1 \mathrm{kHz}$ ). The authors found that temporal discrimination improved with practice. Furthermore, they employed a pretest session before the training phase and a posttest session after the training phase to assess whether this learning would generalize to intervals

R. Ulrich, rolf.ulrich@uni-tuebingen.de 
marked by tones of a different frequency (e.g., $4 \mathrm{kHz}$ ) or to intervals of different durations $(50,200$, or $500 \mathrm{msec})$. It is interesting to us that they found that perceptual learning generalized to the same interval marked by a different frequency only - but not to different interval durations.

Similar results were obtained in a subsequent perceptual learning study by Karmarkar and Buonomano (2003). These authors used a single stimulus protocol. Accordingly, subjects listened to a standard interval several times at the beginning of a single block. After this familiarization phase, subjects were presented with only the comparison tone in each trial. They had to decide whether this comparison was shorter or longer than the standard that was presented during the familiarization phase. One group of subjects was trained with a $100-\mathrm{msec}$ interval that was marked by beeps of $1 \mathrm{kHz}$, whereas another group of subjects was trained with a $200-\mathrm{msec}$ interval that was marked by the same beeps. As in the study by Wright et al. (1997), perceptual learning generalized to stimuli of the same duration as the trained interval, but not to stimuli of a different duration.

In another study on temporal perceptual learning (Nagarajan, Blake, Wright, Byl, \& Merzenich, 1998), subjects performed a $2 \mathrm{AFC}$ task on tactile stimuli presented to one of their hands during the training phase. For one group, the duration of the training stimulus was $75 \mathrm{msec}$, and it was $125 \mathrm{msec}$ for the other group. These authors assessed transfer effects of perceptual learning between different skin locations and different stimulus durations. They also investigated whether there would be a learning transfer from the somatosensory to the auditory system. They reported a complete transfer effect of temporal discrimination to untrained skin locations, as well as a partial transfer to durations that were close to the trained durations, but they found no transfer to distant time intervals $(225 \mathrm{msec})$. A cross-modal learning transfer from the somatosensory to the auditory modality was also observed, but only for an interval that was similar to the trained somatosensory interval. These results are consistent with the idea of an intervalbased timing mechanism that is unaffected by the nontemporal features - such as frequency, amplitude, or even modality - of a given temporal stimulus to be processed (cf. Allan \& Kristofferson, 1974; Rammsayer, 1994).

One aim of the present study was to examine the generality of the findings reviewed above. For this purpose, intramodal and cross-modal transfer effects of training on an auditory temporal discrimination task were assessed. Specifically, subjects were trained with an empty auditory interval of $100 \mathrm{msec}$ (AE100) that was marked by two beeps. We tested whether training effects obtained with this interval would generalize to an empty auditory interval of $500 \mathrm{msec}$ (AE500) and to a filled auditory interval of $100 \mathrm{msec}$ (AF100). The latter stimulus differed from the trained condition in both the interval type (filled vs. empty) and stimulus type (noise vs. tone). Thus, discrimination performance with this filled interval assessed learning transfer to both of these untrained dimensions. The second and more important goal of the present study was to assess whether perceptual training in the AE100 condition would transfer to another sensory modality - that is, to an empty interval of $100 \mathrm{msec}$, with visual markers (VE100). Similar to the design of previous studies (Karmarkar \& Buonomano, 2003; Nagarajan et al., 1998; Wright et al., 1997), potential transfer effects of perceptual learning were assessed by a standard pretest-training-posttest design.

In contrast to previous studies, ${ }^{1}$ and in order to assess potential learning effects occurring during pretest and posttest sessions, we also included a control group that performed only the pretest and posttest phases. This group allowed differentiation between an improvement that resulted from the training phase and learning associated with the pretest and posttest phases. In contrast to previous studies, in order to prevent a possible selection bias, we did not exclude from data analysis subjects who showed no learning effects. Besides wanting to prevent such a bias, we suspected that subjects who showed no overt change in discrimination performance during the learning phase might still benefit from latent learning during this phase. This latent perceptual learning should, therefore, manifest later during the final testing phase. Finally, we used an especially sensitive statistical method (ANCOVA; Vickers, 2001) to assess potential transfer effects.

\section{METHOD}

\section{Subjects}

The trained group consisted of 14 female and 4 male volunteers (mean age $=24.8$ years, $S D=3.7$ ) who participated in one testing session per day for 9 days: two pretest sessions, five practice sessions, and two posttest sessions. These subjects were paid $€ 90$ each for their participation. In addition, a control group of 15 female and 3 male volunteers (mean age $=23.4$ years, $S D=2.3$ ) performed only the two pretest and two posttest sessions; they received $€ 40$ each for their participation. In the control group, the time interval between the pretest and posttest sessions was matched to the one of the trained group and was limited to approximately 2 weeks. All subjects were naive about the purpose of this study, had normal hearing and vision, and had not participated in psychophysical timing experiments before.

\section{Apparatus and Stimuli}

Two types of auditory stimuli were used: filled intervals of white noise (AF100), and empty intervals marked by 15 -msec beeps presented at $1 \mathrm{kHz}$ (AE100, AE500). ${ }^{2}$ Auditory stimuli were presented binaurally via headphones at an intensity of $73 \mathrm{~dB}$ SPL. The visual stimulus in condition VE100 was an empty interval marked by two 15 -msec light pulses $\left(48 \mathrm{~cd} / \mathrm{m}^{2}\right)$, which were produced by a green LED with a diameter of $0.48^{\circ}$. The length of an empty interval was defined as the time interval from the offset of the first marker to the onset of the second marker, whereas the length of a filled interval was defined as the interval between stimulus onset and its offset.

\section{Procedure}

The procedure was similar to that in previous perceptual learning studies on temporal discrimination (Karmarkar \& Buonomano, 2003; Nagarajan et al., 1998; Wright et al., 1997). On each trial, two stimuli of the same type were presented successively. The second stimulus occurred after a random interstimulus interval, which was sampled from a normal distribution with a mean of $1 \mathrm{sec}$ and a standard deviation of $0.1 \mathrm{sec}$. One stimulus represented the standard interval, and the other represented the comparison interval. The presentation order of these two stimuli was randomly determined for each trial. The duration $t$ of the standard was kept constant throughout a single block of trials, whereas the comparison interval was always longer than $t$ - that is, $t+\Delta t$, with $\Delta t>0$. At the end of each trial, subjects had to press one of two designated keys of a computer 
keyboard to indicate whether the first or second interval appeared longer. They then received visual feedback indicating whether the response was correct or incorrect. After $2 \mathrm{sec}$, a new trial started. The instructions to the subjects emphasized accuracy over speed.

The duration of comparison intervals changed according to a weighted up-down adaptive rule (Kaernbach, 1991). In brief, correct answers decreased the comparison interval by a certain downward step, whereas an incorrect answer increased the comparison by a certain upward step. For auditory stimuli, an upward step size corresponded to $1 \%$ of the standard duration, and a downward step corresponded to $3 \%$ of the standard duration. Up and down step sizes for visual stimuli were $4 \%$ and $12 \%$ of the standard, respectively. For both modalities, step sizes were chosen on the basis of the results from a pilot study and were kept constant throughout the experiment. The initial duration of the comparison interval was $120 \mathrm{msec}$ for the 100-msec auditory standard, $140 \mathrm{msec}$ for the 100 -msec visual standard, and $600 \mathrm{msec}$ for the 500 -msec auditory standard. A single block comprised a series of 100 trials, and the data from this series were used to estimate the DL, the $75 \%$ difference threshold in relation to the respective standard duration (cf. Lapid, Ulrich, \& Rammsayer, 2008).

As in the study by Karmarkar and Buonomano (2003), we employed two pretest sessions administered on consecutive days and two posttest sessions, which were also conducted on consecutive days, whereas the first posttest session was conducted the day after the last training session. Each session was composed of eight blocks of trials: two blocks for each of the four stimulus conditions (AE100, AF100, AE500, VE100). All four conditions occurred in the first half of a single session and were repeated in reverse order in the second half of the session. For all subjects, the first and last blocks of each testing session were AE100 (i.e., the training condition). For example, for 1 subject, this order might be as follows: AE100-VE100-AE500AF100-break-AF100-AE500-VE100-AE100. The order of the three other stimulus conditions was counterbalanced according to a Latin square, across all subjects. A single session consisted of 800 trials and lasted approximately $75 \mathrm{~min}$. For each subject, the structure of the two posttest sessions was the same as that of the two pretest sessions.

Each subject of the trained group performed five training sessions (one per day) that started after the pretest sessions and ended before the posttest sessions. The training session contained six blocks of 100 trials each of condition AE100 and lasted about an hour. This amount of daily training has been shown to be sufficient for perceptual learning to take place for temporal discrimination with auditory stimuli (Wright \& Sabin, 2007). In fact, Wright and Sabin showed 360 trials per session to be enough to induce perceptual learning, although previous studies employed more than 600 trials during a single training session (Karmarkar \& Buonomano, 2003, used 720 trials; Nagarajan et al., 1998, used 900-960 trials; Wright et al., 1997, used 900 trials). The first posttest session was administered the day after the subject performed the last training session.

\section{RESULTS}

First, we examined whether the trained group and the control group would exhibit learning in discrimination performance across all sessions. For this analysis, the Weber fraction (DL/standard duration) was computed for condition AE100 for each daily session. The overall mean of the initial Weber fraction in the AE100 condition was $.184(S E M=.014)$ in the first pretest session. Weber fractions of about the same magnitude have been reported for unpracticed subjects by other studies (e.g., Karmarkar \& Buonomano, 2003; Rammsayer \& Lima, 1991; Wright et al., 1997). Figure 1 depicts mean Weber fractions, as a function of session for both the trained group and the control group. A one-way repeated measures ANOVA

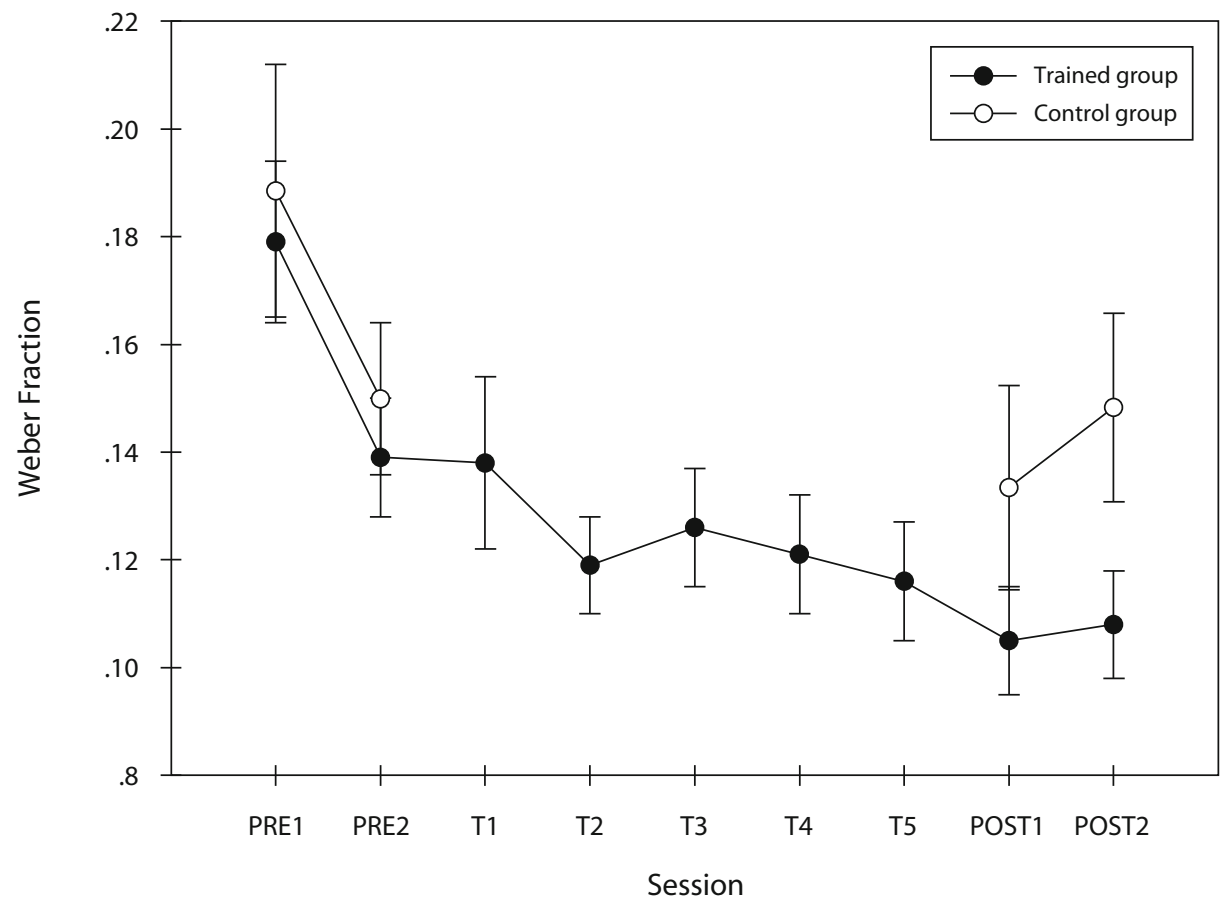

Figure 1. Mean Weber fractions for both the trained and control groups, and for condition AE100 (auditory empty 100-msec interval), as a function of session (pretest phase, training phase, posttest phase). The error bars represent $\pm 1 S E M$. Note that PRE1 and PRE2 refer to the two pretest sessions, whereas POST1 and POST2 correspond to the two posttest sessions. T1, T2, T3, T4, T5: the five training sessions for the trained group. 
confirmed a significant effect of session for the trained group $\left[F(8,136)=9.5, p<.01, \eta^{2}=.36\right]$. In addition, trend analysis revealed a significant linear component $[F(1,136)=57.32, p<.01]$, as well as a significant quadratic component $[F(1,136)=9.04, p<.01]$. Focusing only on the training sessions (Sessions $3-7$ ), there is still a significant linear trend $[F(1,68)=4.08, p<.05]$. These results confirm that discrimination performance improved with training, and thus they are consistent with the results of the previous studies mentioned in the introduction. An analogous ANOVA for the control group in the AE100 condition indicated a significant improvement across the four sessions $\left[F(3,51)=5.58, p<.01, \eta^{2}=.25\right]$. The mean Weber fraction again revealed a significant linear trend $[F(1,17)=8.02, p<.05]$ and a significant quadratic component $[F(1,17)=5.43, p<.05]$.

Figure 1 indicates that learning was already present during the pretest phase. A three-way ANOVA [group (trained, control) $\times$ pretest day (first, second) $\times$ condition (AE100, AF100, AE500, VE100)] on the Weber fraction was conducted to examine this issue in more detail. There was a significant main effect of pretest day $[F(1,34)=$ $\left.41.9, p<.01, \eta^{2}=.55\right]$. The mean Weber fraction was $.202(\mathrm{SE}=.010)$ on the first day and $.173(\mathrm{SE}=.008)$ on the second day. In addition, there was a significant main effect of condition $\left[F(3,102)=96.2, p<.01, \eta^{2}=.81\right]$. Consistent with findings in previous studies on temporal discrimination (e.g., Ulrich et al., 2006), the Weber fraction was larger for the visual than for the auditory modality. In particular, the mean fractions were $.164(\mathrm{SE}=$ $.011)$ for AE100, $148(\mathrm{SE}=.005)$ for AF100, $.109(\mathrm{SE}=$ $.006)$ for AE500, and .329 (SE $=.020)$ for VE100. Finally, there was a significant interaction of condition and pretest day $\left[F(3,102)=3.5, p=.017, \eta^{2}=.40\right]$, because the Weber fraction decreased not uniformly from the first to the second day for the four conditions. Specifically, the decrease was .039, .009, .019, and .051 for AE100, AF100, AE500, and VE100, respectively. No further effect was significant. An analogous ANOVA was performed for the 2 posttest days to see whether learning was still present during the posttest phase. The mean Weber fraction was $.156(\mathrm{SE}=.007)$ on the first posttest day and .163 $(\mathrm{SE}=.010)$ on the second posttest day $[F(1,34)=1.5$, $\left.p=.233, \eta^{2}=.04\right]$. In addition, factor posttest day did not significantly interact with condition $[F(3,102)=1.3$, $\left.p=.264, \eta^{2}=.04\right]$. These two results suggest that learning did not further improve temporal discrimination during the posttest phase. There was also a significant main effect of condition $\left[F(3,102)=92.5, p<.01, \eta^{2}=.73\right]$. The mean Weber fractions in the posttest phase were .122 $(\mathrm{SE}=.010)$ for AE100, $.136(\mathrm{SE}=.004)$ for $\mathrm{AF} 100, .089$ $(\mathrm{SE}=.007)$ for AE500, and .291 ( $\mathrm{SE}=.020)$ for VE100. No further significant effects were obtained.

Figure 2 shows the mean Weber fractions for each condition and each group, for the pretest phase and the posttest phase. For each condition, statistical analysis was performed on the average pretest thresholds (measured over the first 2 days) and the average posttest thresholds (measured over the last 2 days). Each subsequent analysis assessed potential changes between the pretest and

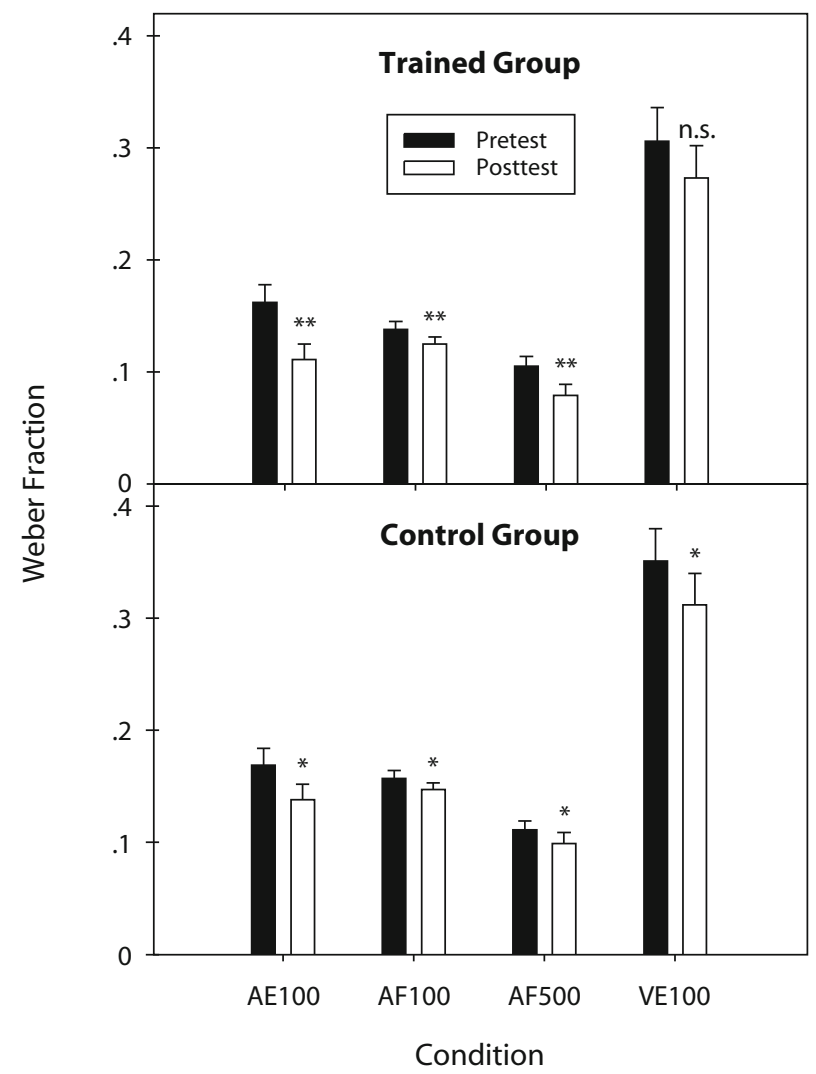

Figure 2. Mean Weber fractions, as a function of condition and test, using the averaged Weber fractions of both days from the pretests and the averaged Weber fractions of both days from the posttests. The upper panel depicts the results for the trained group, and the lower panel depicts the results for the control group. The error bars represent 1 SEM. A significant change between pretest and posttest scores is indicated for each condition and group (one-tailed $t$ test). ${ }^{*} p<.05 .{ }^{* *} p<.01$.

posttest measures for the two groups and for each single condition. If the training phase increased the preexisting performance level for the trained group relative to that for the control group, performance changes should be larger for the trained group. Such a comparison can be made in several ways (see Vickers, 2001). ${ }^{3}$ Here we employed an ANCOVA because this procedure adjusts for baseline differences in performance, and it generally has a greater statistical power than other methods for measuring changes between pretest and posttest scores (see Vickers, 2001; Vickers \& Altman, 2001). This analysis can be described by a single regression equation, $W_{2}=c+a \cdot W_{1}+b \cdot$ group, where $W_{1}$ and $W_{2}$ are the Weber fractions of a single subject (measured during the pretest and posttest phases, respectively), $c$ is a constant, $a$ and $b$ are regression coefficients, and group is coded " 1 " for the control group and "0" for the trained group. Thus, the pretest score is used as the covariate to assess the main effect of group (trained vs. control) on the posttest scores as the dependent measure. This regression equation adjusts the posttest measure $W_{2}$ of each subject for his or her pretest measure $W_{1}$ and thus yields the result of whether or not there is some imbalance in the pretest scores. Of major 
interest is the coefficient $b$, because it reflects potential performance differences between the two groups. More precisely, we expected $b$ to be significantly larger than zero if discrimination performance in the trained group benefited from training.

A separate ANCOVA was conducted for each condition (AE100, AF100, AE500, VE100; see Figure 3). As expected, the estimate of $b$ (i.e., $b_{\text {est }}=.024$ ) was significantly larger than zero in condition AE100 $[t(33)=1.66$, $p=.05]$. Thus, when subjects were trained in condition AE100, they performed better in the posttest phase than when they were not trained in this condition. Theoretically more important, however, an analogous analysis revealed that the training phase induced specific transfer effects. First, the estimate of $b\left(b_{\text {est }}=.011\right)$ was significantly larger than zero in condition AF100 $[t(33)=1.89, p<$ $.05]$. This improvement can be attributed to a transfer effect resulting from training on the AE100 condition. Sec-
AE100

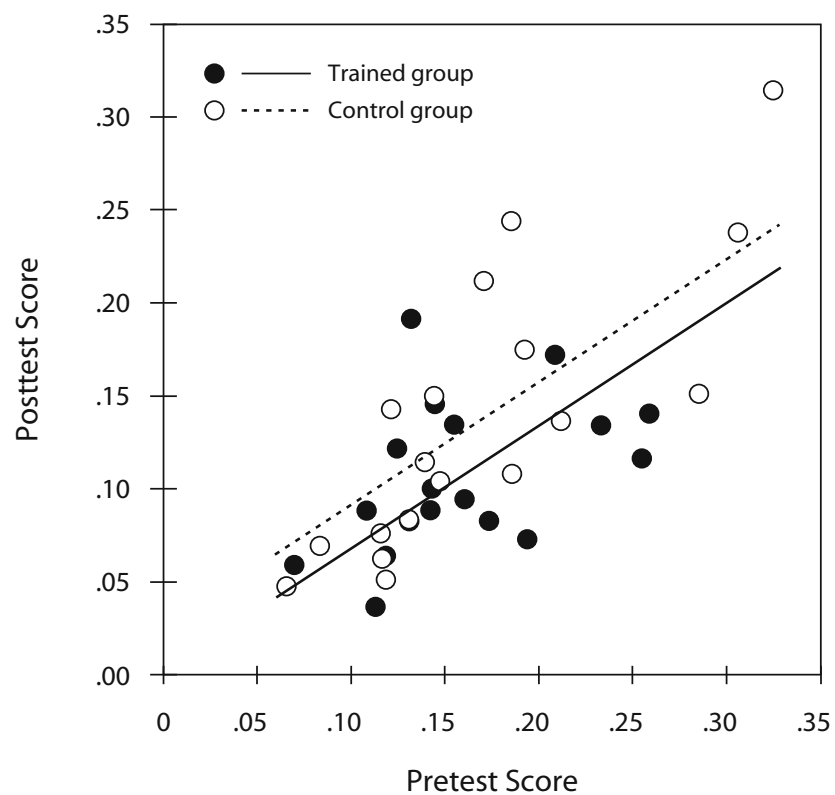

AE500

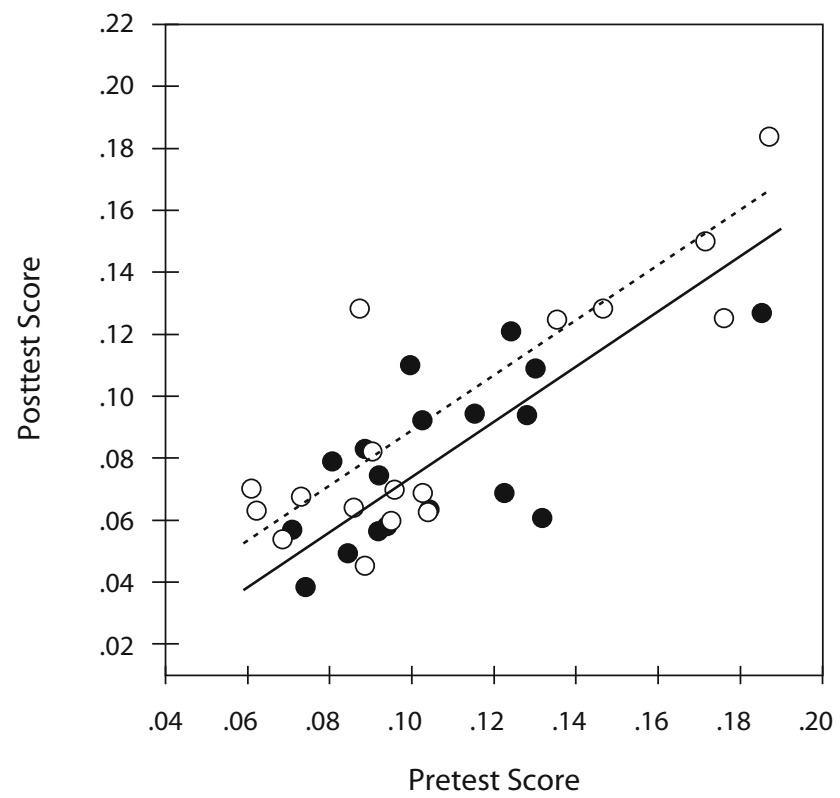

AF100

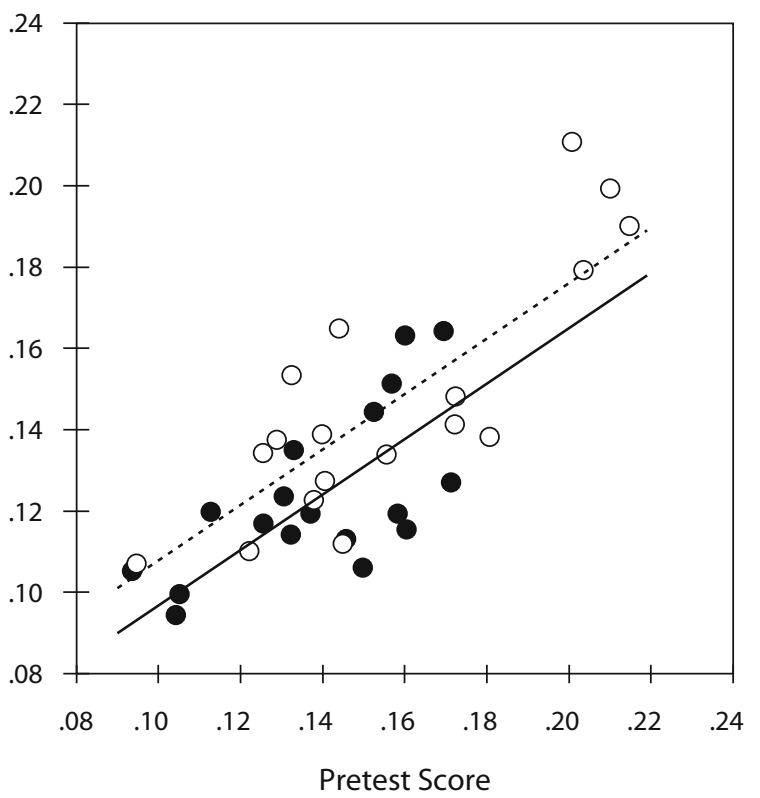

VE100

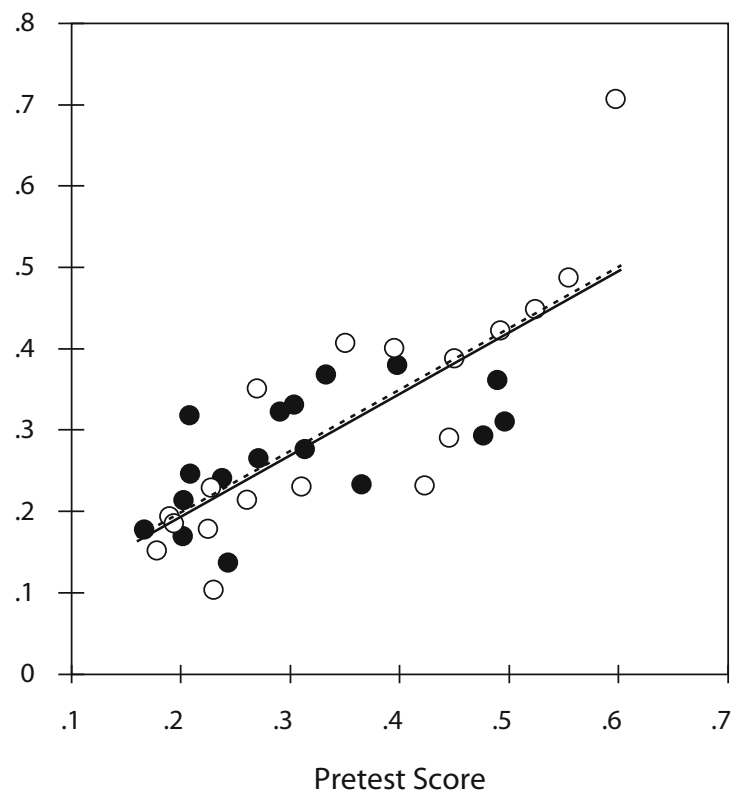

Figure 3. Pretest and posttest scores in each condition, and fitted lines. The estimated difference between the trained group and the control group from the ANCOVA is the vertical distance between the two lines. AE100, auditory empty 100-msec interval; AF100, auditory filled 100-msec interval; AE500, auditory empty 500-msec interval; VE100, visual empty 100-msec interval. 
ond, a similar transfer effect was obtained for condition AE500 $\left(b_{\text {est }}=.015\right)[t(33)=1.78, p<.05]$. Finally, the analysis yielded no significant transfer effect for condition VE100 $\left(b_{\text {est }}=.005\right)[t(32)=0.21, p=.42]$. In sum, then, the analyses showed specific transfer effects from training with condition AE100 to conditions AF100 and AE500, but no significant cross-modal transfer effect to condition VE100. ${ }^{4}$

\section{DISCUSSION}

The present study employed a perceptual learning paradigm to examine potential cross-modal transfer effects in a $2 \mathrm{AFC}$ duration-discrimination task. Stated more precisely, the primary goal was to assess whether training on auditory duration discrimination would facilitate visual duration discrimination. Visual discrimination performance was not better after training in the trained group than it was after training in the control group, who had received no extensive training in condition AE100. This result indicates that, under the circumstances tested here, no cross-modal transfer effect occurred from the auditory to the visual modality.

A secondary goal of our study was to replicate previously reported transfer effects within the auditory modality. First, as in the Karmarkar and Buonomano (2003) study, subjects in the trained group significantly improved their discrimination performance in the untrained AF100 condition. This is consistent with Karmarkar and Buonomano's hypothesis that nontemporal features of the auditory signal do not influence transfer effects within the auditory modality within the same interval duration. This finding also agrees with the other reports of transfer effects across different auditory frequencies (for auditory training, see Karmarkar \& Buonomano, 2003; Wright et al., 1997 ) and across different skin locations (for somatosensory training, see Nagarajan et al., 1998). Second, and in contrast to previous findings (Karmarkar \& Buonomano, 2003; Wright et al., 1997), our subjects also exhibited an improvement of temporal discrimination in the untrained AE500 condition. In the remainder of this discussion, we will compare the present findings with those reported in the literature.

First, our data appear not to be consistent with those of Nagarajan et al. (1998), which indicated a cross-modal transfer effect. Nagarajan et al. reported that temporal discrimination of tactile stimuli with a duration of $125 \mathrm{msec}$ improved temporal discrimination of auditory stimuli when the trained and untrained stimuli in both modalities were close in duration (125 vs. $100 \mathrm{msec})$, but not when they were disparate in duration ( $125 \mathrm{vs} .50 \mathrm{msec}$ or $125 \mathrm{vs}$. $200 \mathrm{msec}$ ). They concluded from this result that learning in the somatosensory modality transfers to the auditory one, and that this transfer is interval specific. Unfortunately, however, their control group did not include auditory conditions. For this reason, it remains unclear whether this improvement in their auditory condition merely reflects learning occurring during the pretest and posttest phases or reflects true transfer learning.
Second, we could only partially replicate the withinmodality effects on temporal discrimination for the auditory modality that were reported by Wright et al. (1997) and Karmarkar and Buonomano (2003). Similar to what was seen in previous work, we also observed a significant improvement for filled intervals. Specifically, the untrained condition AF100 showed a significant improvement in the trained group relative to the control group. However, we did not observe an absence of the transfer effect for the untrained, 500-msec, empty interval, as did Wright et al. It is hard to see why we observed this significant improvement in the AE500 condition but Wright et al. and Karmarkar and Buonomano (2003) did not. Perhaps the null effect obtained in those previous studies reflects a Type II error, due to the smaller sample sizes employed in those studies, as compared with our sample size.

The present data do not support a cross-modal training transfer of temporal discrimination performance from the auditory to the visual modality. There are at least two explanations for this result. First, one may assume that each sensory modality possesses its own timing mechanism that is primarily involved in the discrimination of brief durations (e.g., Bendixen et al., 2005; Rammsayer \& Lima, 1991). If perceptual learning improves temporal processing within such a sensory-specific mechanism, it is quite conceivable that cross-modal transfer effects cannot arise. Nevertheless, this notion proceeds from the existence of range-specific timers, an assumption that does not receive support from the present data or from other work (e.g., Rammsayer \& Ulrich, 2005).

Second, visual signals are usually judged to be shorter than auditory ones, even when both signals are of equivalent duration (e.g., Behar \& Bevan, 1961; Goldstone \& Goldfarb, 1964; Goldstone \& Lhamon, 1974; Penney, Gibbon, \& Meck, 2000; Wearden, Edwards, Fakhri, \& Percival, 1998). Therefore, it is possible that learning did not generalize from the auditory to the visual system in the present study because AE100 and VE100 differed in perceived duration (yet the same perceived duration might be necessary for transfer). This account could explain the present lack of a transfer effect from audition to vision. With regard to this account, however, it is difficult to see why a transfer occurred from the short to the long duration (i.e., from AE100 to AE500) within the auditory modality.

Although the present study does not support a transfer from audition to vision, Meegan, Aslin, and Jacobs (2000) found that training on an auditory perceptual task generalized to a motor task, thereby reducing motor timing variability on the trained interval. These authors suggested a common neural architecture underlying analysis of sensory input and control of motor output. A lack of transfer from audition to vision in the present experiment is not in conflict with those findings. Perhaps timing effects within a sensory modality are strongly coupled with motor timing because the two systems share common timers (see, e.g., Ivry \& Hazeltine, 1995).

The lack of a cross-modal transfer from audition to vision is surprising because previous work (Guttman, Gilroy, \& Blake, 2005) suggests that a visual temporal 
structure is transformed into an auditory representation. Specifically, Guttman et al. have put forward the hypothesis that people encode visual temporal sequences automatically and involuntarily in the form of an auditory code. In addition, they have proposed the possibility of multimodal cortex activation that contains a mechanism for supporting generalized temporal processing. As a result, one might expect to find improvement of temporal discrimination in the visual modality, even though practicing takes place within the auditory modality. It is worth noting, however, that Guttman et al. did not examine temporal discrimination performance, but examined primarily memory performance, for remembering visual temporal sequences. Hence, the present data $d o$ contradict their findings. Nevertheless, their findings point to the possibility of an asymmetric transfer from the visual to the auditory modality, but not vice versa, since the auditory modality dominates temporal information processing (see, e.g., Fendrich \& Corballis, 2001; Recanzone, 2003; Repp \& Penel, 2002). This asymmetric processing of temporal information would be difficult to reconcile with an amodal pacemaker-counter mechanism that receives direct input from different sensory modalities (see, e.g., Ulrich et al., 2006). In addition, the hypothesis by Guttman et al. suggests the idea that one should observe a visual-to-auditory training transfer, but not-as shown in this study - an auditory-to-visual one. Future research is necessary to address this point.

In conclusion, then, in the present study, we investigated whether perceptual learning of temporal discrimination in the auditory modality would transfer to the visual modality. The present data do not support such a crossmodal transfer effect. In addition, the present study was only partially successful in replicating within-modality transfer patterns of temporal discrimination. Furthermore, the present data suggest that the performance increment of the trained group, relative to the performance increment of the control group, represents a genuine training effect. Finally, we feel that future research needs to include controls without training and should use sensitive statistical techniques (Vickers, 2001).

\section{AUTHOR NOTE}

This work was supported by the Deutsche Forschungsgemeinschaft (UL 116/8-3). We thank Andy Kramer and Marie-Luise Zeitler for their help in data collection, and also Daniel Bratzke, Karin Bausenhart, J. Devin McAuley, and two anonymous reviewers for constructive comments. Correspondence concerning this article should be addressed to R. Ulrich, Department of Psychology, University of Tübingen, Friedrichstr. 21, 72072 Tübingen, Germany (e-mail: rolf.ulrich@uni-tuebingen.de).

\section{REFERENCES}

Allan, L. G., \& Kristofferson, A. B. (1974). Psychophysical theories of duration discrimination. Perception \& Psychophysics, 16, 26-34.

Behar, I., \& BEVAN, W. (1961). The perceived duration of auditory and visual intervals: Cross-modal comparison and interaction. American Journal of Psychology, 74, 17-26.

Bendixen, A., Grimm, S., \& Schröger, E. (2005). Human auditory event-related potentials predict duration judgments. Neuroscience Letters, 383, 284-288

Buhusi, C. V., \& Meck, W. H. (2005). What makes us tick? Functional and neural mechanisms of interval timing. Nature Reviews Neuroscience, 6, 755-764.

Fendrich, R., \& Corballis, P. M. (2001). The temporal cross-capture of audition and vision. Perception \& Psychophysics, 63, 719-725.

Goldstone, S., \& GoldFarb, J. L. (1964). Auditory and visual time judgment. Journal of General Psychology, 70, 369-387.

Goldstone, S., \& Lhamon, W. T. (1974). Studies of auditory-visual differences in human time judgment: I. Sounds are judged longer than lights. Perceptual \& Motor Skills, 39, 63-82.

Grondin, S. (2003). Studying psychological time with Weber's Law. In R. Buccheri, M. Saniga, \& W. M. Stuckey (Eds.), The nature of time: Geometry, physics, and perception (pp. 33-41). Dordrecht, The Netherlands: Kluwer.

Guttman, S. E., Gilroy, L. A., \& Blake, R. (2005). Hearing what the eyes see: Auditory encoding of visual temporal sequences. Psychological Science, 16, 228-235.

Huang, C.-B., Zhou, Y. F., \& Lu, Z. L. (2008). Broad bandwidth of perceptual learning in the visual system of adults with anisometropic amblyopia. Proceedings of the National Academy of Sciences, 105, 4068-4073.

Ivry, R. B., \& Hazeltine, R. E. (1995). Perception and production of temporal intervals across a range of durations: Evidence for a common timing mechanism. Journal of Experimental Psychology: Human Perception \& Performance, 21, 3-18.

Ivry, R. B., \& SPENCER, R. M. C. (2004). The neural representation of time. Current Opinion in Neurobiology, 14, 225-232.

JoNES, M. R. (1976). Time, our lost dimension: Toward a new theory of perception, attention, and memory. Psychological Review, 83, 323355 .

Jones, M. R., \& Boltz, M. (1989). Dynamic attending and responses to time. Psychological Review, 96, 459-491.

KAERNBACH, C. (1991). Simple adaptive testing with the weighted updown method. Perception \& Psychophysics, 49, 227-229.

Karmarkar, U. R., \& BUONOMANo, D. V. (2003). Temporal specificity of perceptual learning in an auditory discrimination task. Learning \& Memory, 10, 141-147.

Karmarkar, U. R., \& Buonomano, D. V. (2007). Timing in the absence of clocks: Encoding time in neural network states. Neuron, 53, 427-438.

Lapid, E., Ulrich, R., \& Rammsayer, T. [H.] (2008). On estimating the difference limen in duration discrimination tasks: A comparison of the 2AFC and the reminder task. Perception \& Psychophysics, 70, 291-305.

LARGe, E. W., \& Jones, M. R. (1999). The dynamics of attending: How people track time-varying events. Psychological Review, 106, 119159.

LEWIS, P. A., \& Miall, R. C. (2003). Distinct systems for automatic and cognitively controlled time measurement: Evidence from neuroimaging. Current Opinion in Neurobiology, 13, 250-255.

Mauk, M. D., \& Buonomano, D. V. (2004). The neural basis of temporal processing. Annual Review of Neuroscience, 27, 307-340.

McAuley, J. D., \& Jones, M. R. (2003). Modeling effects of rhythmic context on perceived duration: A comparison of interval and entrainment approaches to short-interval timing. Journal of Experimental Psychology: Human Perception \& Performance, 29, 1102-1125.

Meegan, D. V., Aslin, R. N., \& Jacobs, R. A. (2000). Motor timing learned without motor training. Nature Neuroscience, 3, 860-862.

Nagarajan, S. S., Blake, D. T., Wright, B. A., Byl, N., \& MerzeNICH, M. M. (1998). Practice-related improvements in somatosensory interval discrimination are temporally specific but generalize across skin location, hemisphere, and modality. Journal of Neuroscience, 18, 1559-1570.

Penney, T. B., Gibbon, J., \& Meck, W. H. (2000). Differential effects of auditory and visual signals on clock speed and temporal memory. Journal of Experimental Psychology: Human Perception \& Performance, 26, 1770-1787.

RAMMSAYER, T. H. (1994). Effects of practice and signal energy on duration discrimination of brief auditory intervals. Perception \& Psychophysics, 55, 454-464.

RAMMSAYeR, T. H., \& Lima, S. D. (1991). Duration discrimination of filled and empty auditory intervals: Cognitive and perceptual factors. Perception \& Psychophysics, 50, 565-574. 
RAMmSAYER, T. [H.], \& UlRICH, R. (2001). Counting models of temporal discrimination. Psychonomic Bulletin \& Review, 8, 270-277.

RAmmsayer, T. [H.], \& UlRich, R. (2005). No evidence for qualitative differences in the processing of short and long temporal intervals. Acta Psychologica, 120, 141-171.

Recanzone, G. H. (2003). Auditory influences on visual temporal rate perception. Journal of Neurophysiology, 89, 1078-1093.

Repp, B. H., \& Penel, A. (2002). Auditory dominance in temporal processing: New evidence from synchronization with simultaneous visual and auditory sequences. Journal of Experimental Psychology: Human Perception \& Performance, 28, 1085-1099.

Ulrich, R., NitschKe, J., \& RAMmsaYer, T. [H.] (2006). Crossmodal temporal discrimination: Assessing the predictions of a general pacemakercounter model. Perception \& Psychophysics, 68, 1140-1152.

VICKERS, A. J. (2001). The use of percentage change from baseline as an outcome in a controlled trial is statistically inefficient: A simulation study. BMC Medical Research Methodology, 1, 6-9.

Vickers, A. J., \& Altman, D. G. (2001). Analysing controlled trials with baseline and follow up measurements. British Medical Journal, 323, 1123-1124.

Wearden, J. H., Edwards, H., Fakhri, M., \& Percival, A. (1998). Why "sounds are judged longer than lights": Application of a model of the internal clock in humans. Quarterly Journal of Experimental Psychology, 51B, 97-120.

Wright, B. A., Bounomano, D. V., Mahncke, H. W., \& Merzenich, M. M. (1997). Learning and generalization of auditory temporalinterval discrimination in humans. Journal of Neuroscience, 17, 3956-3963.

Wright, B. A., \& Sabin, A. T. (2007). Perceptual learning: How much daily training is enough? Experimental Brain Research, 180, 727736.

\section{NOTES}

1. Only the study of Nagarajan et al. (1998) included a control group of 6 subjects who received no training. Interestingly, not only their trained group but also their control group showed smaller thresholds for the posttest than for the pretest phase. However, this difference was not significantly different from zero for the control group. This nonsignificant result might reflect a Type II error. In fact, the reported standard errors were particularly large for this group (see Nagarajan et al., 1998, Figure 5A). In order to augment the statistical power in our study, we increased the size of the control group to $n=18$.

2 . The empty temporal interval in this study was measured from the offset of the first beep to the onset of the second beep. By contrast, the empty interval in Wright et al. (1997) and Nagarajan et al. (1998) was measured from the onset of the first marker to the onset of the second marker.

3. Some studies of perceptual learning (e.g., Huang, Zhou, \& Lu, 2008; Nagarajan et al., 1998) have used the percentage of fractional change to measure changes in performance. This measure is defined as fractional change $=\left(W_{1}-W_{2}\right) / W_{1} \cdot 100 \%$, where $W_{1}$ and $W_{2}$ represent the Weber fraction in the pretest phase and posttest phase, respectively. This measure compares the change in performance relative to the initial base level. Nagarajan et al. (1998, p. 1561) suggested that perceptual learning is present when fractional change differs significantly from zero for the trained group. Applying this definition to the data of our trained group, the mean fractional changes were 30.0, 9.7, 25.0, and $7.5 \%$ in conditions AE100, AF100, AE500, and VE100, respectively. One-tailed $t$ tests showed a significant fractional change for conditions $\mathrm{AE} 100[t(17)=4.68, p<.01], \mathrm{AF} 100[t(17)=3.35, p<.01]$, and AE500 $[t(17)=5.79, p<.01]$, but not for condition VE100 $[t(17)=$ 1.26]. Thus, according to Nagarajan et al.'s criterion, one might conclude that training on AE100 transfers to AF100 and AE500, but not to VE100. Unfortunately, however, the same conclusion would apply to the control group. For the control group, the mean fractional changes were 19.4, 5.0, 11.7, and $11.3 \%$ in conditions AE100, AF100, AE500, and VE100, respectively. A one-sided $t$ test suggested a significant change for AE100 [t(17) $=3.21, p<.01]$, for AF100 $[t(17)=1.69, p<.05]$, for AE500 $[t(17)=1.92, p<.05]$, and even for VE100 $[t(17)=2.22$, $p<.05]$. The result of the control group clearly shows that this definition is not sufficient to evaluate potential transfer effects. Therefore, one may examine whether the fractional change is larger in the trained than in the control group in order to provide a stricter test for the presence of perceptual learning. This stricter test revealed only a significant increase in fractional change for condition AE500 [ $t(34)=1.77, p<.05]$, but not for the remaining conditions. This null result may simply reconfirm the conclusion that fractional change is a statistically insensitive measure (Vickers, 2001).

4. We repeated an ANCOVA using only the scores obtained from the second pretest and the first posttest day. Although this analysis confirmed the results reported in the main text, just the beta coefficient $b$ in condition AF100 proved to be significantly larger than zero. This result has to be attributed to the reduced statistical power of this analysis, because mean Weber fractions in this analysis are based on only $50 \%$ of the data points, as compared with the analysis reported in the main text. Therefore, the reliability of these scores in the additional analysis is expected to be lower than the one for the full data set. We performed a reliability analysis to examine this point. Cronbach's alpha was .84 for the pretest scores when the data of the first and second pretest days were used to compute the pretest scores. By contrast, Cronbach's alpha decreased to .75 when only the data points of the second day were employed for these scores. Likewise, alpha was equal to .89 when the posttest scores were based on the data of the first and second posttest days, but alpha reduced to .84 when only the data of the first posttest day were used. It is also remarkable that the scores in condition VE100 were relatively reliable. Cronbach's alpha was .88 for the pretest scores (based on both pretest days) in this condition, and .89 for the posttest scores (based on both posttest days). Thus, the lack of a transfer effect to condition VE100 cannot be attributed to a particularly low reliability.

(Manuscript received October 23, 2007; revision accepted for publication September 10, 2008.) 